\title{
POLYNOMIALS OF BEST APPROXIMATION ON AN INFINITE INTERVAL*
}

BY

\author{
JAMES M. EARL
}

Introduction. This paper is concerned with an extension of certain studies of the degree of convergence of methods of approximation to a given function. The approximating functions are polynomials determined so as to minimize the integral over an infinite interval of the product of a non-negative weight function and the $m$ th power of the magnitude of the error. The essential point of this discussion is that the interval of integration is infinite. The case of a finite interval has been considered by Jackson $\dagger$ and others for polynomials as well as for other types of approximating functions.

The problem may be formulated more precisely as follows. Let $f(x)$ be a given function of $x$ defined on an infinite interval $S$. Under suitable restrictions on $f(x)$ and the non-negative weight function $r(x)$, there exists a determination of the coefficients in the polynomial

$$
p_{n}(x)=a_{0}+a_{1} x+a_{2} x^{2}+\cdots+a_{n} x^{n}
$$

for which the integral

$$
\int_{S} r(x)\left|f(x)-p_{n}(x)\right|^{m} d x
$$

is a minimum. When the coefficients in $p_{n}(x)$ are so determined, the polynomial $p_{n}(x)$ is called an approximating polynomial of degree $n$ and is denoted by $P_{m n}(x)$ while the value of the minimized integral is denoted by $I_{m n}$. The problem is to investigate the degree of the uniform convergence of $P_{m n}(x)$ to $f(x)$ when $m$ is a fixed positive number and $n$ is allowed to become infinite.

The Hermite series and the Laguerre series are two important special cases of the least-square problem, $m=2$, on the doubly infinite interval $(-\infty$, $\infty)$ and the simply infinite interval $(0, \infty)$ respectively. The former corresponds to the weight function $r(x)=\exp \left(-x^{2}\right)$ and the latter to $r(x)=\exp$

\footnotetext{
* Presented to the Society, April 7, 1928; received by the editors in May, 1930.

$\dagger$ See D. Jackson, On the convergence of certain trigonometric and polynomial approximations, these Transactions, vol. 22 (1921), pp. 158-166; Note on the convergence of weighted trigonometric series, Bulletin of the American Mathematical Society, vol. 29 (1923), pp. 259-263; and bibliographical references there noted.
} 
$(-x)$. In these special cases, the problem of uniform convergence has been extensively studied by various methods. ${ }^{*}$ The method used here consists of obtaining an expression which gives an upper bound for the error in the approximation to $f(x)$ in terms of the integral $I_{m n}$ and then of obtaining upper bounds for $I_{m n}$ to be substituted in this expression. By this method, results on uniform convergence are obtained for very general exponential weight functions, for the doubly infinite as well as for the simply infinite interval, and for any given positive $m$. While these results possess considerable generality, they are intended to suggest rather than to exhaust the wide range of applicability of the method.

The arrangement of the paper is in summary as follows. After a statement of well known theorems for reference in $\S 1$, the questions of the existence and the uniqueness of the approximating polynomials are considered in $\$ 2$. The upper bounds for the magnitude of the error $f(x)-P_{m n}(x)$ obtained in $\$ 3$ involve $I_{m n}$; the question of degree of convergence is thus reduced to the problem of determining upper bounds for the integral $I_{m n}$. This is done in $\S 4$ for the case of an essentially even weight function on the doubly infinite interval; the upper bound for $I_{m n}$ obtained depends not only on the nature of $f(x)$ but on the behavior of $r(x)$ for large values of $|x|$. Application to the special case of an exponential weight function $r(x)$ is made in $\S 5$. The case of a simply infinite interval taken for definiteness as $(0, \infty)$ is made to depend on the above in $\S 6$ and it is found that the necessary restriction on $r(x)$ for large values of $|x|$ is less for the simply than for the doubly infinite interval. Section 7 contains a method for the treatment of essentially skew weight functions on the doubly infinite interval. It is shown in the application to an exponential weight function that the necessary restriction on $r(x)$ for large positive values of $x$ can be weakened at the expense of increasing the restriction on $r(x)$ for large negative values of $x$. Unification of the results of $\S \S 3$ to 6 is incidentally effected in $\S 7$.

1. Theorems for reference. The theorems due to S. Bernstein, $\nmid$ A. Markoff, $\ddagger$

* For bibliography, see E. Hille, A class of reciprocal functions, Annals of Mathematics, (2) vol. 27 (1926), pp. 427-464. See also W. E. Milne, On the degree of convergence of the Gram-Charlier series, these Transactions, vol. 31 (1929), pp. 422-433; the concluding section of Chapter III in the colloquium lectures of D. Jackson, The Theory of Approximation, New York, 1930; and an abstract by J. M. Earl, The degree of convergence of Hermite and Laguerre series, Bulletin of the American Mathematical Society, vol. 35 (1930), p. 455.

$\dagger \mathrm{S}$. Bernstein, Sur l'ordre de la meilleure approximation des fonctions continues par des polynomes de degré donné, Mémoire couronné, Brussels, 1912.

$\ddagger$ For proof see M. Riesz, Eine trigonometrische Interpolationsformel $\cdots$, Jahresbericht der Deutschen Mathematiker-Vereinigung, vol. 23 (1914), pp. 354-368. 
P. L. Tchebycheff, ${ }^{*}$ and D. Jackson, $\uparrow$ respectively, that follow are lettered for convenience of reference.

In Theorems B, M and T below, let $q_{n}(x)$ with derivative $q_{n}^{\prime}(x)$ be any polynomial of degree $n$ which satisfies the stated hypothesis.

Theorem B. If $\left|q_{n}(x)\right| \leqq L$ on $(a, b)$, then $\left|q_{n}^{\prime}(x)\right| \leqq n L /[(b-x)(x-a)]^{1 / 2}$ on $(a, b)$.

Theorem M. If $\left|q_{n}(x)\right| \leqq L$ on $(a, b)$, then $\left|q_{n}^{\prime}(x)\right| \leqq 2 n^{2} L /(b-a)$ on $(a, b)$.

Theorem T. (a) If $\left|q_{n}(x)\right| \leqq L$ on $(-a, a)$, then $\left|q_{n}(x)\right| \leqq L|2 x / a|^{n}$ when $x$ is not on $(-a, a)$.

(b) If $\left|q_{n}(x)\right| \leqq L$ on $(0, a)$ [or on $\left.(-a, 0)\right]$, then $\left|q_{n}(x)\right| \leqq L|4 x / a|^{n}$ on $(a, \infty)[$ or on $(-\infty,-a)]$.

THEOREM J. If on the interval $(a, b), f(x)$ has a pth derivative $f^{(p)}(x)$ which satisfies a Lipschitz condition of order $u$ with coefficient $L$, that is

$$
\left|f\left(x_{2}\right)-f\left(x_{1}\right)\right| \leqq L\left|x_{2}-x_{1}\right|^{u} \quad(0<u \leqq 1)
$$

for every pair of values $x_{1}, x_{2}$ on $(a, b)$, then for each integer $n>p$, there exists $a$ polynomial $p_{n}(x)$ of degree $n$ such that

$$
\left|f(x)-p_{n}(x)\right| \leqq K L[(b-a) / n]^{p+u}
$$

uniformly on $(a, b)$ where $K$ is an absolute constant.

2. The existence and the uniqueness of the approximating polynomials. The first lemma below may be used as the basis for proofs of existence of approximating functions in a variety of least $m$ th power problems. A similar lemma has been proved for bounded $\S h$ 's. The functions $h_{1}(x), \cdots, h_{n}(x)$ are called properly independent on a point set $S$ if every linear combination of these functions in which the coefficients are not all zero is different from zero on a sub-set of $S$ of positive measure. The set $S$ may be thought of as bounded or not bounded in this section.

\footnotetext{
* For proof see S. Bernstein, Leçons sur les Propriétés Extrémales et la Meilleure Approximation des Fonctions Analytiques d'une Variable Rélle, Paris, 1926, pp. 7-8.

$\dagger$ See, for example, D. Jackson, On the approximate representation of an indefinite integral ... , these Transactions, vol. 14 (1913), Theorem VIII, p. 354.

$\ddagger$ D. Jackson, $A$ generalized problem in weighted approximation, these Transactions, vol. 26 (1924), pp. 133-154; pp. 133-137.

$\$$ Shohat has removed the restriction of boundedness in a special case for polynomial approximation; see J. Shohat, On the polynomial of best approximation to a given continuous function, Bulletin of the American Mathematical Society, vol. 31 (1925), pp. 509-514; p. 510.
} 
Lemma 1. Suppose that the functions $h_{1}(x), \cdots, h_{n}(x)$ are properly independent on $S$ and that the integral

$$
J=\int_{S}\left|c_{1} h_{1}(x)+\cdots+c_{n} h_{n}(x)\right|^{m} d x
$$

exists for each set of coefficients $\left(c_{1}, \cdots, c_{n}\right)$. Then there exists a positive constant $A$ which is independent of the c's such that

$$
\left|c_{k}\right| \leqq(J / A)^{1 / m} \quad(k=1, \cdots, n) .
$$

The generality of the hypothesis that the integral $J$ exists for each set of coefficients is apparent on noticing that this hypothesis is satisfied for example when each $h$ is measurable and has a summable $m$ th power on $S$. The proof of Lemma 1 follows.

Let $C$ denote the set of points $\left(c_{1}, \cdots, c_{n}\right)$ in $n$ dimensions such that $c_{k}=1$ for some $k$ and $\left|c_{k}\right| \leqq 1$ for all $k=1, \cdots, n$. Corresponding to each point of $C$, the integral $J$ is positive. The set $C$ is closed. Therefore since $J$ is a continuous function of $c_{1}, \cdots, c_{n}$, bounded for all points of $C$, there exists a least $J=A$ which is positive.

Now let the $c$ 's in $J$ be arbitrary. The lemma is true if the $c$ 's are all zero. Suppose then that some $c$, say $c_{k}$, is not zero and has at least as great magnitude as any other coefficient. Then the coefficients in the quotient $\left[c_{1} h_{1}(x)\right.$ $\left.+\cdots+c_{n} h_{n}(x)\right] / c_{k}$ are a point of $C$ so that $J /\left|c_{k}\right|^{m} \geqq A$ and from this follows the inequality (2).

THEOREM 1. Suppose that for each polynomial $p_{n}(x)$ of degree $n$, the integral (1) exists and that $\int_{S} r(x) d x=R>0$.

(a) If $m$ is positive, at least one approximating polynomial $P_{m n}(x)$ exists.

(b) If $m$ exceeds unity, the approximating polynomial $P_{m n}(x)$ is unique.

(c) If $m=1$ and $f(x)$ is continuous, the approximating polynomial $P_{m n}(x)$ is unique.

Statement (a) of this theorem follows from Lemma 1 by the usual reasoning. The usual proof of (b) applies here while (c) may be proved by extending a proof given by Shohat* for the case of a finite interval $S$. These proofs are omitted.

3. The degree of convergence of the approximating polynomials. Here $(a, b)$ denotes a finite interval which is a sub-set of the set $S$ which may be thought of as bounded or not bounded. If $r(x)$ satisfies Hypothesis $\mathrm{Y}$ (given below) on this interval, then upper bounds in terms of $n$ and $I_{m n}$ for $\mid f(x)-$ $P_{m n}(x) \mid$ on $(a, b)$ and $(a+\epsilon, b-\epsilon)$ are given below by expressions (9) and (10)

* J. Shohat, loc. cit., pp. 511-513. 
respectively where $\epsilon$ is any preassigned positive constant less than $\frac{1}{2}(b-a)$. In the proofs, $U$ denotes the least upper bound of $|f(x)|$ on $(a, b)$ and it is assumed that $\left|P_{m n}(x)\right|$ attains its maximum value $\pi_{j}$ on $(a+j \epsilon / k, b-j \epsilon / k)$ at $x=x_{i}(j=0,1, \cdots, k), k$ being any given positive integer. Various constants which are independent of $n$ are denoted by the single letter $c$. The minimized integral $I_{m n}$ is of course taken over the whole of $S$.

Lемма 1. If $r(x)$ has a positive lower bound $v$ on $(a, b)$ and if $\pi_{j}>4 U$, then

$$
\pi_{j} \leqq c\left(n I_{m n} \pi_{j-1}\right)^{1 /(m+1)} \quad(j=1, \cdots, k) .
$$

By Theorem B, $\left|P_{m n}^{\prime}(x)\right| \leqq(k n / \epsilon) \pi_{j-1}$ on $(a+j \epsilon / k, b-j \epsilon / k)$. It follows by the law of the mean that

$$
\left|P_{m n}(x)-P_{m n}\left(x_{j}\right)\right| \leqq(k n / \epsilon) \pi_{j-1}\left|x-x_{j}\right| \leqq \frac{1}{2} \pi_{j}
$$

whenever $\left|x-x_{j}\right| \leqq \pi_{j}\left(2 k n \pi_{j-1} / \epsilon\right)^{-1}$ so that $\left|P_{m n}(x)\right| \geqq \frac{1}{2} \pi_{j}$ on an interval $w$ of length $c \pi_{j} /\left(n \pi_{j-1}\right)$ which lies wholly on $(a+j \epsilon / k, b-j \epsilon / k)$ and has $x_{j}$ for one end point. It follows that

$$
\left|f(x)-P_{m n}(x)\right| \geqq\left|P_{m n}(x)\right|-|f(x)| \geqq \frac{1}{2} \pi_{j}-\frac{1}{4} \pi_{j}=\frac{1}{4} \pi_{j}
$$

on $w$ and, since $r(x) \geqq v$ on $w$, that

$$
I_{m n} \geqq c \pi_{j}^{m} \int_{w} r(x) d x \geqq c \pi_{j}^{m+1} /\left(n \pi_{j-1}\right) .
$$

The inequality (3) of the lemma results on solution of this inequality for $\pi_{j}$.

Hypothesis $\mathrm{Y}$. The function $r(x)$ is said to satisfy Hypothesis $\mathrm{Y}$ on $(a, b)$ if there exist positive constants $D, d$ and non-negative constants $g$, $s$ such that $r(x)$ has a positive lower bound $v=D d^{g+s}$ on $(a, b)$ except perhaps on $(l+2)$ non-overlapping sub-intervals $(a, a+d),\left(y_{i}-d, y_{i}+d\right)(i=1, \cdots$, $l)$ and $(b-d, b)$ on which $r(x)$ behaves as follows:

$$
\begin{aligned}
& r(x) \geqq D(b-x)^{\circ} \quad \text { whenever } b-x \leqq d, \\
& r(x) \geqq D(x-a)^{\circ} \quad \text { whenever } x-a \leqq d, \\
& r(x) \geqq D\left|x-y_{i}\right|^{\text {s }} \text { whenever }\left|x-y_{i}\right| \leqq d .
\end{aligned}
$$

Under Hypothesis $\mathrm{Y}$, the weight function may become zero in a restricted manner at a finite number of interior points as well as at the end points of $(a, b)$. If, for example, $S$ is the interval $(0, \infty)$ and $(a, b)$ is $(0, A)$, then using the upper bound (9) of Theorem 2 for the error, it is seen that there is no weakening in the degree of convergence provable caused by allowing the weight function to become zero at a finite number of interior points of $(0, A)$ provided the order of vanishing in no case exceeds $s=1$. The essential novelty of the convergence proofs given here lies in the difference in the effect of 
allowing $r(x)$ to vanish to a given order at interior points of $(a, b)$ as compared with the effect of allowing $r(x)$ to vanish to the same order at one or both end points of $(a, b)$.

In Lemmas 2 and 4 below, $(\alpha, \beta)$ denotes any given interval which is interior to $(a, b)$ and which contains the intervals $\left(y_{i}-d, y_{i}+d\right)(i=1, \cdots, l)$.

Lemмa 2. Suppose that $r(x)$ satisfies Hypothesis $\mathrm{Y}$ on $(a, b)$ and that $\pi_{0}>4 U$. Then

$$
\begin{aligned}
& \pi_{0} \leqq c\left(n^{s+1} I_{m n}\right)^{1 / m} \text { if } x_{0} \text { is on }(\alpha, \beta), \\
& \pi_{0} \leqq c\left(n^{2(o+1)} I_{m n}\right)^{1 / m} \text { if } x_{0} \text { is on }(a, \alpha) \text { or on }(\beta, b) .
\end{aligned}
$$

If $x_{0}$ is on $(\alpha, \beta)$, then by Theorem $\mathrm{B},\left|P_{m n}^{\prime}(x)\right| \leqq c n \pi_{0}$ on $(\alpha, \beta)$. Steps similar to those used in the proof of Lemma 1 except that $w$ is here replaced by $w^{\prime}$ of length $1 /(2 c n)$ with $x_{0}$ as one end point and that $r(x)$ which is replaced by $v$ in relation (4) is here replaced by $D\left|x_{0}-x\right|^{*}$, result in inequality (5).

If $x_{0}$ is on $(a, \alpha)$ [or on $(\beta, b)$ ], Theorem M gives $\left|P_{m n}^{\prime}(x)\right| \leqq c n^{2} \pi_{0}$. The proof of (6) is similar to the proof of (5) but $w^{\prime}$ is here replaced by $w^{\prime \prime}$ of length $1 /\left(2 c n^{2}\right)$ and $s$ is replaced by $g$.

The following lemma results on setting $s=g=0$ in inequalities (5) and (6).

LEMma 3. If $r(x)$ has a positive lower bound on $(a, b)$ and if $\pi_{0}>4 U$, then $\pi_{0} \leqq c\left(n^{2} I_{m n}\right)^{1 / m}$.

Lemma .4. Suppose that $r(x)$ satisfies Hypothesis $\mathrm{Y}$ on $(a, b)$ and that $\pi_{k}>4 U$. Then

$$
\pi_{k} \leqq c\left(n^{8+1} I_{m n}\right)^{1 / m}
$$

if $x_{k}$ is on $(\alpha, \beta)$,

$$
\pi_{k} \leqq c\left(n^{\boldsymbol{B}} I_{m n}\right)^{1 / m}
$$

where $H=1+(m+1)^{-k}$, if $x_{k}$ is on $(a+\epsilon, \alpha)$ or on $(\beta, b-\epsilon)$.

If $x_{k}$ is on $(\alpha, \beta)$, inequality (7) is obtained in the same manner as (5) of Lemma 2. If $x_{\varepsilon}$ is on $(a+\epsilon, \alpha)$, then when $\epsilon$ is sufficiently small, $r(x)$ has a positive lower bound on $\left(a+\frac{1}{2} \epsilon, \alpha+\frac{1}{2} \epsilon\right)$. Let $\pi_{0}^{\prime}$ be the maximum of $\left|P_{m n}(x)\right|$ on this interval. By Lemma $3, \pi_{0}^{\prime} \leqq c\left(n^{2} I_{m n}\right)^{1 / m}$ so that the inequality (8) results by successive applications of Lemma 1 with $(a, b)$ replaced by $\left(a+\frac{1}{2} \epsilon, \alpha+\frac{1}{2} \epsilon\right)$ and $\epsilon$ by $\frac{1}{2} \epsilon$. This establishes Lemma 4 .

If the magnitude of the error in the approximation to $f(x)$ on $(a, b)$ by a polynomial $q_{n}(x)$ does not exceed $\epsilon_{n}$, then $U$ for the function $f(x)-q_{n}(x)$ does 
not exceed $\epsilon_{n}$ and, since, by Lemma 2 , the approximating polynomial $P_{m n}(x)$ $-q_{n}(x)$ for this function does not exceed $4 \epsilon_{n}$ plus the sum of the expressions on the right in inequalities (5) and (6), the magnitude of $f(x)-P_{m n}(x)$ does not exceed the expression (9) on $(a, b)$. Similar reasoning using Lemma 4 instead of Lemma 2 results in the upper bound (10) of Theorem 2 for the error on $(a+\epsilon, b-\epsilon)$.

THEOREM 2. Suppose that $f(x)$ can be uniformly approximated by a polynomial of degree $n$ with an error which nowhere exceeds $\epsilon_{n}$ on the interval $(a, b)$. Let $\epsilon$ be any positive number less than $\frac{1}{2}(b-a)$. If $r(x)$ satisfies Hypothesis $\mathrm{Y}$ on $(a, b)$, then $\left|f(x)-P_{m n}(x)\right|$ does not exceed

$$
\begin{gathered}
5 \epsilon_{n}+c\left[\left(n^{2(\theta+1)}+n^{s+1}\right) I_{m n}\right]^{1 / m} \text { on }(a, b), \\
5 \epsilon_{n}+c\left[\left(n^{B}+n^{s+1}\right) I_{m n}\right]^{1 / m} \text { on }(a+\epsilon, b-\epsilon),
\end{gathered}
$$

where $H=1+(m+1)^{-k}$.

4. The order of magnitude of the minimized integral. An upper bound for the integral $I_{m n}$ is obtained here for the case of an even weight function $r(x)$. Extensions to the case of more general weight functions are then suggested. The set $S$ is taken to be the doubly infinite interval on which $r(x)$, as well as the product of $r(x)$ and the $m$ th power of each of the functions $x^{\alpha} M(x)(\alpha>0)$ and $\left|f(x)-p_{n}(x)\right|$, is assumed to be summable where $p_{n}(x)$ is any polynomial of degree $n$ and $M(x)$ is even, non-negative, non-decreasing for non-negative values of $x$ and such that on every interval $(-a, a)$, the $p$ th derivative $f^{(p)}(x)$ of $f(x)$, assumed to exist, satisfies a Lipschitz condition of order $u$ with coefficient $M(a)$. In addition to the above, let it be assumed throughout that $f(x)$ and its first $p$ derivatives are zero when $x=0$; this condition may always be realized by adding to $f(x)$ a polynomial of degree $p$ and since the approximations to $f(x)$ are by polynomials of degree $n>p$, there is no loss of generality in the assumption.

It will be convenient to denote by $G_{M r}(a, A), H_{M r}(a, A), K_{n}$, and $V_{p}(x, \alpha)$ the following expressions where $A \geqq a>0$ and where $q_{n}(x)$ denotes a polynomial of degree $n$ :

$$
\begin{aligned}
G_{M r}(a, A) & =\left[M(a) A^{p+u}\right]^{m}\left[n^{-m(p+u)}+\int_{A}^{\infty} r(x)(2 x / A)^{m n} d x\right], \\
H_{M r}(a, A) & =G_{M r}(a, A)+\int_{a}^{\infty} r(x)\left[M(x) x^{p+u}\right]^{m} d x, \\
K_{n} & =\int_{-\infty}^{\infty} r(x)\left|f(x)-q_{n}(x)\right|^{m} d x, \\
V_{p}(x, \alpha) & =f(\alpha)+f^{\prime}(\alpha)(x-\alpha)+\cdots+f^{(p)}(\alpha)(x-\alpha)^{p} / p ! .
\end{aligned}
$$


The various constants denoted hereafter by $c$ are independent not only of $n$ but of $A, a$ and $M(x)$.

In Lemmas 1 to 3 , let it be assumed that $r(x)$ is an even function of $x$. The following lemma results by successive integrations from 0 to $x$.

LeMma 1. For every real value of $x,\left|f^{(p-j)}(x)\right| \leqq M(x)|x|^{i+u}(j=0,1$, $\cdots, p)$.

LEMMA 2. If $M(x)=M$ is constant, then for every integer $n>p$, there exists a polynomial $q_{n}(x)$ of degree $n$ such that $K_{n}$ does not exceed $c G_{M r}(a, A)$.

By Theorem J, there exists a polynomial $q_{n}(x)$ such that

$$
\left|f(x)-q_{n}(x)\right| \leqq c M(A / n)^{p+u}
$$

on $(-A, A)$. Moreover, $|f(x)|$ does not exceed $M A^{p+u}$ on $(-A, A)$, by Lemma 1 , so that for points on $(-A, A)$ by (11) and therefore for points not on $(-A, A)$ by Theorem $\mathrm{T},\left|q_{n}(x)\right|$ does not exceed $c M A^{p+u}$ and $c M A^{p+u}|2 x / A|^{n}$ respectively. By Lemma $1,|f(x)|$ nowhere exceeds $M A^{p+u}|x / A|^{p+u}$. Therefore, since $(p+u)$ does not exceed $n$,

$$
\left|f(x)-q_{n}(x)\right| \leqq c M A^{p+u}|2 x / A|^{n}
$$

when $x$ is not on $(-A, A)$ because then $|x / A|$ exceeds unity. Since $r(x)$ is even, the lemma results on replacing the error $\left|f(x)-q_{n}(x)\right|$ in $K_{n}$ by its upper bounds (11) and (12) on and not on $(-A, A)$ respectively.

In the following lemma, the restriction that $M(x)$ be constant is removed.

Lemma 3. For every integer $n>p$, there exists a polynomial $q_{n}(x)$ of degree $n$ such that $K_{n}$ does not exceed $c H_{M r}(a, A)$.

The auxiliary function $g(x)$ which is defined as $f(x), V_{p}(x, a)$ and $V_{p}(x,-a)$ when $x$ is on $(-a, a),(a, \infty)$ and $(-\infty,-a)$ respectively satisfies the conditions of Lemma 2 with $M=M(a)$, so that there exists a polynomial $q_{n}(x)$ such that the first integral on the right in the inequality

$$
K_{n} \leqq 2^{m} \int_{-\infty}^{\infty} r(x)\left|g(x)-q_{n}(x)\right|^{m} d x+2^{m} \int_{-\infty}^{\infty} r(x)|f(x)-g(x)|^{m} d x
$$

does not exceed $c G_{M r}(a, A)$. Moreover, by Lemma 1, neither $|f(x)|$ nor $|g(x)|$ exceeds $M(x)|x|^{p+u}$ and therefore since the integrand in the second integral on the right in (13) is zero on $(-a, a)$, this integral does not exceed a constant multiple of the last term in the expression for $H_{M r}(a, A)$. This establishes Lemma 3. 
The following theorem is an immediate consequence of Lemma 3 because the integral $I_{m n}$ never exceeds $K_{n}$.

THEOREM 3. If $S$ is the doubly infinite interval and if $r(x)$ is an even function of $x$, then for every integer $n>p$,

$$
I_{m n} \leqq c H_{M r}(a, A) .
$$

The significance of the various parameters entering into the somewhat complicated expression $H_{M_{r}}(a, A)$ will be made clearer as the applications of the above theorem are worked out in detail.

Now, to what related classes of weight functions may the theory of this section be extended? This is a question which naturally arises because it is sometimes desirable to use results for a simple class of weight functions in obtaining results for other classes of weight functions. No complete answer to the question is given here. It may be pointed out, however, that the upper bound for $I_{m n}$ in Theorem 3 is not increased as to order of magnitude when $r(x)$ is replaced by a constant multiple of $r(x)$, or more generally by the product of $r(x)$ and a non-negative bounded function of $x$; the convergence of the error $f(x)-P_{m n}(x)$ toward zero, on the other hand, may be retarded when the new weight function vanishes to a higher order than the original weight function $r(x)$. In the proof of Corollary 3 of the next section, it is noted that the magnitude of $I_{m n}$ is essentially unchanged even when the exponential weight function $r(x)$ is replaced by $|\pi(x)| r(x)$ where $\pi(x)$ is any given polynomial. While the problem of the extension of simple classes of weight functions is important, the case of an arbitrary weight function may be treated independently as in $\$ 7$ of this paper.

5. The exponential weight function. Application of $\$ 4$ to particular functions $r(x)$ and $M(x)$ involves two important steps illustrated respectively by Lemmas 1 and 2 below. In the first step, $A$ in the expression for $H_{M r}(a, A)$ is chosen as a function of $n$, the choice depending only on $r(x)$. This, by Theorem 3, gives an upper bound for $I_{m n}$ in terms of $n$ and $a$ which is chosen in the second step as a function of $n$. The upper bound for $I_{m n}$ thus obtained may then be substituted for $I_{m n}$ in Theorem 2, giving, as in Theorem 4 below, an upper bound for the error in the approximation to $f(x)$ by $P_{m n}(x)$ in terms of $n$.

The discussion of this section refers to the weight function $r(x)=\exp$ $\left(-h|x|^{\sigma}\right)(h>0, \sigma>1)$ and to $M(x)=\exp \left(j|x|^{\sigma}\right)(0 \leqq m j<h)$, except where explicit notation to the contrary is made. Notice that the exponent of $|x|$ in both $r(x)$ and $M(x)$ is the same number $\sigma$; Corollaries 1 and 3 state results which apply to $M(x)=\exp (d|x| i)(d \geqq 0)$ where $i$ is less than $\sigma$. 
LeMma 1. The number $A$ can be chosen as a constant multiple of $n^{1 / \sigma}$ so that

$$
H_{M r}(a, A) \leqq c\left[M(a) n^{-(\sigma-1)(p+u) / \sigma}\right]^{m}+\int_{a}^{\infty} \exp \left(-h x^{\sigma}\right)\left[M(x) x^{p+u}\right]^{m} d x
$$

The integral from $A$ to $\infty$ in the expression for $H_{M r}(a, A)$ does not exceed the integral of $\exp \left(-\frac{1}{2} h x^{\sigma}\right)$, which is bounded, times the product of $(2 / A)^{m n}$ and the maximum $(2 m n /(h e \sigma))^{m n / \sigma}$ of $x^{m n} \exp \left(-\frac{1}{2} h x^{\sigma}\right)$ on $(0, \infty)$. The lemma therefore results on setting $A$ equal to $h^{1 / \sigma}$ times the constant $4(2 m /(h e \sigma))^{1 / \sigma}$.

LEMMA 2. If $\omega>1$ is assigned, then $I_{m n}$ does not exceed

$$
c n^{-m(\sigma-1)(p+u)(1-m j / h) /(\omega \sigma)}
$$

prosided $n$ is sufficiently great.

Let $v>1$ be chosen so that

$$
c\left(n^{m^{2} j(v-1)(\sigma-1)(p+u) /(h \sigma)}+1\right) n^{-m(\sigma-1)(p+u)(1-m j / h) / \sigma}
$$

is less than one half the expression (16). The lemma follows from Theorem 3 on noticing that the expression on the right in (15) does not exceed (17) and therefore (16), provided $a=[(v / h)(m / \sigma)(\sigma-1) \cdot(p+u) \log n]^{1 / \sigma}$ and prcvided $n$ is so great that the integral from $a$ to infinity in (15) does not exceed $\exp \left(-[h-m j] a^{\sigma} / v\right)$ which does not exceed one half the expression (16).

Theorem 4. Let $\rho$ be any assigned positive number less than 1 and let $(\alpha, \beta)$ be any given finite interval. Then for $n$ sufficiently great, the error $\mid f(x)$ $-P_{m n}(x) \mid$ does not exceed

$$
c n^{-\rho(p+u)(1-m j / h)(\sigma-1) / \sigma+1 / m} \text { on }(\alpha, \beta) .
$$

Let the interval $(a, b)$ in Theorem 2 be $(\alpha-\epsilon, \beta+\epsilon)$; then $g=s=0$. Under the hypothesis on $f(x), 5 \epsilon_{n}$ may be taken, by virtue of Theorem $\mathrm{J}$, as $c n^{-(p+u)}$, so that Theorem 4 results on substitution of the expression (16) for $I_{m n}$ in (10) with $(\omega-1)$ and $(1 / k)$ taken sufficiently small.

It is perhaps worth while to dwell at some length on the implications of Theorem 4; the specializations and extensions of this theorem included below are suggestive of the wide range of applicability of the methods of this paper.

Notice first that uniform convergence results whenever the exponent of $n$ in (18) is negative; it is necessary for this that $\sigma$ exceed 1 . When $m$ is positive and $\sigma$ does exceed 1 , then uniform convergence does always result provided $f(x)$ is restricted by taking $p$ sufficiently great. It will be seen in the next section that when the doubly infinite interval is replaced by a simply 
infinite interval, then it is necessary only that $\sigma$ exceed $\frac{1}{2}$ instead of 1 ; this difference in restriction on $\sigma$ is compromised, in $\$ 7$, in the case of a skew weight function.

Next consider the most simple case, $j=0$, in which $f^{(p)}(x)$ satisfies an ordinary Lipschitz condition on $(-\infty, \infty)$ with a constant coefficient. Under these circumstances, a sufficient condition for uniform convergence is that $(p+u)(\sigma-1) / \sigma>1 / m$. This is also a sufficient condition for uniform convergence when $f^{(p)}(x)$ is considerably less restricted for large values of $|x|$ by taking $M(x)=\exp \left(d|x|^{i}\right)(d \geqq 0, i<\sigma)$; this results because the expression (18) need not be altered in form when $M(x)$ is replaced by a new function which nowhere exceeds a constant multiple of $M(x)$. In particular, if $j>0$ is assigned, then there exists a positive constant $\gamma$ such that the new function $M(x)=\exp \left(d|x|^{i}\right)(d \geqq 0, i<\sigma)$ nowhere exceeds $\gamma \exp \left(j|x|^{\sigma}\right)$. In fact, the following corollary results when $\rho<1$ and $j>0$ in (18) are assigned so that $\rho(1-m j / h)=\phi$.

Corollary 1. Suppose that $r(x)=\exp \left(-h|x|^{\sigma}\right)$ and that $M(x)=\exp$ $(d|x| i)(d \geqq 0, i<\sigma)$. Let $\phi$ be any assigned positive number less than 1 and let $(\alpha, \beta)$ be any given finite interval. Then for $n$ sufficiently great, the error $\left|f(x)-P_{m n}(x)\right|$ does not exceed $c n^{-\phi(p+u)(\sigma-1) / \sigma+1 / m}$ on $(\alpha, \beta)$.

The following corollary restates the results of Theorem 4 and Corollary 1 for the special case of the Hermite series in which $r(x)=\exp \left(-x^{2}\right)$ and $m=2$.

CoRollary 2. Let $\rho$ be any assigned positive number less than 1. Then the error in the approximation to $f(x)$ on any given finite interval by the nth partial sum of the Hermite series does not exceed

$$
\begin{aligned}
c n^{-(1 / 2) \rho(p+u)(1-2 j)+1 / 2} \text { when } M(x) & =\exp \left(j x^{2}\right) & (0<j<1), \\
c n^{-(1 / 2) \rho(p+u)+1 / 2} \text { when } M(x) & =\exp \left(d|x|^{i}\right) & (d \geqq 0, i<2) .
\end{aligned}
$$

A sufficient condition for uniform convergence on any given finite interval of the Hermite series when $M(x)=\exp \left(\frac{1}{4} x^{2}\right)$ for example, is that $p \geqq 2$, and a sufficient condition under the more restrictive hypothesis $M(x)=\exp \left(d|x|^{i}\right)$ $(i<2)$ is that $p \geqq 1$.

Consider now an extension, of the type mentioned in $\$ 4$, of the results for the weight function $r(x)=\exp \left(-h|x|^{\sigma}\right)$. Let $r(x)=\exp \left(-h|x|^{\sigma}\right)$ be replaced by $r(x)=|\pi(x)| \exp \left(-h|x|^{\sigma}\right), \pi(x)$ being any given polynomial. For this more general weight function, the expression (16) still gives an upper bound for the integral $I_{m n}$ and on any finite interval which contains no root of $\pi(x)$, the statements of all results on uniform convergence given for the weight function $\exp \left(-h|x|^{\sigma}\right)$ remain unaltered. Moreover, any interval 
$(\alpha, \beta)$ on which $\pi(x)$ does vanish, may be enclosed in an interval $(a, b)$ on which $r(x)$ satisfies Hypothesis $\mathrm{Y}$ with $g=0$ and with $s$ equal to the greatest order of vanishing of $\pi(x)$ on $(\alpha, \beta)$. The upper bound for the error in the approximation given in the following corollary is obtained by use of the expression (10) of Theorem 2 because an upper bound for the error on $(a, b)$ is an upper bound for the error on the sub-interval $(\alpha, \beta)$ of $(a, b)$.

Corollary 3. Suppose that $r(x)=|\pi(x)| \exp (-h|x| \sigma) \quad(h>0, \sigma>1)$ where $\pi(x)$ is a polynomial having no root of order greater than $s$ on the given interval $(\alpha, \beta)$ and that $M(x)=\exp (d|x| i)(d \geqq 0, i<\sigma)$. Let $\rho$ be any assigned positive number less than 1 . Then

$$
\left|f(x)-P_{m n}(x)\right| \leqq c n^{-\rho(p+u)(\sigma-1) / \sigma+(s+1) / m}
$$

uniformly on $(\alpha, \beta)$.

The result of this corollary corresponds to the result of Corollary 1; a result corresponding to Theorem 4 , which may be readily stated, is left for the reader.

Remark. When the weight function $r(x)$ tends toward zero very rapidly when $|x|$ becomes infinite, the effect on the magnitude of $I_{m n}$ of the interval $S=(-\infty, \infty)$ being infinite is less pronounced. For example, if $r(x)=\exp$ ( $\left.-\exp x^{2}\right)$ and $M(x)=\exp (\exp \cdot|x| i)(i<2)$, then $A$ and $a$ may be chosen, in the steps illustrated by Lemmas 1 and 2 of this section, as constant multiples of $(\log \cdot n)^{1 / 2}$ and $(\log \log n)^{i / 2}$ respectively so that $I_{m n}$ does not exceed $c n^{-m(p+u) / \omega}$ and hence, by Theorem 2, the error $\left|f(x)-P_{m n}(x)\right|$ does not exceed $c n^{-\rho(p+u)+1 / m}$ where $\rho$ and $(1 / \omega)$ are positive numbers less than 1 . For the case of a finite interval $S$ and a weight function having a positive lower bound on $S$, the corresponding upper bounds for $I_{m n}$ and the error on any given interval interior to $S$ are $c n^{-m(p+u)}$ and $c n^{-\rho(p+u)+1 / m}$ respectively. The details in the verification of the above statements are left for the reader.

6. The simply infinite interval. In this section, the minimized integral $I_{m n}$ is over a simply infinite interval $S$, taken for definiteness as $(0, \infty)$, and the functions $r(x), M(x)$ and $f(x)$ satisfy the general hypotheses of $\S 4$ on this interval. In Lemma 1 below, an upper bound for $I_{m n}$ is obtained in terms of an integral, with an even weight function, over the doubly infinite interval in which $f\left(t^{2}\right)$ is the function to be approximated. The integral over the doubly infinite interval may then be bounded as is done in Theorem 5 below by use of Theorem 3 .

LEMMa 1. If $p_{n}(t)$ is any polynomial in $t$ of degree $n$, then

$$
I_{m n} \leqq 2 \int_{-\infty}^{\infty}|t| r\left(t^{2}\right)\left|f\left(t^{2}\right)-p_{n}(t)\right|{ }^{m} d t .
$$


Let $\frac{1}{2}\left[p_{n}(t)+p_{n}(-t)\right]$, which is a polynomial of degree $n$ at most in $t^{2}=x$, be denoted by $q_{n}(x)$. Then $I_{m n}$ does not exceed the integral

$$
\begin{aligned}
& \int_{0}^{\infty} r(x)\left|f(x)-q_{n}(x)\right|^{m} d x \\
= & \int_{-\infty}^{\infty}|t| r\left(t^{2}\right)\left|\frac{1}{2}\left[f\left(t^{2}\right)-p_{n}(t)\right]+\frac{1}{2}\left[f\left(t^{2}\right)-p_{n}(-t)\right]\right|^{m} d t,
\end{aligned}
$$

which in turn does not exceed the expression on the right in inequality (21). This establishes Lemma 1.

The following lemma is a result of Lemma 1 of $\$ 4$ and of the formula for the $p$ th derivative with respect to $t$ of $f\left(t^{2}\right)$. The details of the proof are omitted.

Lемма 2. The pth derivative with respect to $t$ of $f\left(t^{2}\right)$ satisfies a Lipschitz condition of order $u$ with coefficient $c M\left(a^{2}\right) a^{p+u}$ on every interval $-a \leqq t \leqq a$.

Let $p_{n}(t)$ in Lemma 1 be the approximating polynomial for $f\left(t^{2}\right)$ corresponding to the even weight function $\rho(t)=|t| r\left(t^{2}\right)$ on the doubly infinite interval. Then by application of Theorem $3, I_{m n}$ does not exceed $c H_{\mu \rho}(a, A)$ where $\mu(t)=M\left(t^{2}\right)|t|^{p+u}$. The expression (22) is not less than a constant multiple of the expression gotten by putting $x=t^{2}$ in $c H_{\mu \rho}(a, A)$ and then replacing $a^{2}, A^{2}$ by $a, A$ respectively; therefore $I_{m n}$ does not exceed a constant multiple of (22) as stated in the following theorem.

THEOREM 5. If $S$ is the simply infinite interval $(0, \infty)$, the integral $I_{m n}$ does not exceed a constant multiple of

$$
\begin{gathered}
{\left[M(a)(a A)^{(p+u) / 2}\right]^{m}\left[n^{-m(p+u)}+\int_{A}^{\infty} r(x)(4 x / A)^{m n} d x\right]} \\
+\int_{a}^{\infty} r(x)\left[M(x) x^{p+u}\right]^{m} d x .
\end{gathered}
$$

The expression (22) corresponds to $H_{M r}(a, A)$ of Theorem 3. The process of application of these results involves choosing $A$ and $a$ in terms of $n$ so that the integrals from $A$ to infinity and from $a$ to infinity respectively are negligible when $n$ becomes infinite. The non-negligible term of $H_{M r}(a, A)$ is $\left[M(a)(A / n)^{p+u}\right]^{m}$ which is large with respect to the corresponding term $\left[M(a)(a A)^{(p+u) / 2} / n\right]^{m}$ of $(22)$, provided $a$ is small with respect to $A$. For example, let $r(x)=\exp \left(-h|x|^{\sigma}\right)$ and let $M(x)=\exp \left(d|x|^{i}\right)(i<\sigma, h, d \geqq 0)$. Let $A$ and $a$ be chosen as $c n^{1 / \sigma}$ and $c(\log n)^{i / \sigma}$ as in $\S 5$. Then if $S=(0, \infty)$,

$$
\left|f(x)-P_{m n}(x)\right| \leqq c n^{-\rho(p+u)(\sigma-1 / 2) / \sigma+1 / m}
$$


uniformly on any given finite interval $(\alpha, \beta)$ where $\alpha$ and $\rho$ are positive and $\rho$ is less than 1. Uniform convergence results when the exponent of $n$ on the right in inequality (23) is negative and for this it is necessary that $\sigma$ exceed $\frac{1}{2}$ instead of 1 as was the case for the doubly infinite interval with the same $r(x)$ and $M(x)$. The result (23) corresponds to that of Corollary 1 of $\S 5$; comparison of these results shows first that for a given type of $r(x)$ and of $M(x)$, a higher order of uniform convergence is to be expected on the simply than on the doubly infinite interval and second that the types of admissible weight functions for the simply infinite interval include types of weight functions inadmissible on the doubly infinite interval.

Corresponding to each result of $\$ 5$, there is a result for the simply infinite interval gotten by replacing $\sigma$ in $\S 5$ by $2 \sigma$. When $r(x)=\exp (-x)$ on the interval $(0, \infty)$ and $m=2$, for example, the results obtained apply to the Laguerre instead of the Hermite series. Theorems on the degree of uniform convergence on any finite interval including the point $x=0$ may be developed by using expression (9) instead of expression (10) of Theorem 2 in the proofs. The details of these statements and the proofs are left for the reader.

7. Essentially skew weight functions. The integral $I_{m n}$ of this section is over the doubly infinite interval $S$. The discussion is intended to cover the case of essentially skew weight functions such as the exponential weight function discussed after the proof of Theorem 6 below; the discussion is, however, general and effects a unification of the results of $\$ \$ 4$ to 6 . The weight function $r(x)$ is subject only to general conditions of integrability such as those of $\S 4$ but $r(x)$ is not in general an even function of $x$. The function $f(x)$ is assumed throughout to have a $p$ th derivative $f^{(p)}(x)$ which satisfies a Lipschitz condition of order $u$ with coefficient $M(a)$ on $(0, a)$ and with coefficient $M(-a)$ on $(-a, 0)$ for every $a$, where $M(x)$ and $M(-x)$ are non-negative and non-decreasing for non-negative values of $x$. It is assumed as usual that $f(x)$ and its first $p$ derivatives vanish when $x=0$.

Let it be explicitly understood that $B$ is taken as zero, in Lemma 1 or in Theorem 6 of this section, only when $r(x)$ is identically zero for negative values of $x$ and that the integral over $(-\infty,-B)$ in (25) is then zero. With this understanding, if $B=0$, the upper bound for $I_{m n}$ of Theorem 6 reduces essentially to the upper bound of Theorem 5 for the minimized integral over the simply infinite interval. On the other hand, when $r(x)$ and $M(x)$ are even functions of $x$ and $a=b$ and $A=B$, then Theorem 6 gives essentially the upper bound of Theorem 3 for the minimized integral over the doubly infinite interval with an even weight function. Thus, Theorems 3 and 5 are essentially special cases of the more general Theorem 6. 
A skew exponential weight function is used to illustrate the application of Theorem 6; the application follows the proof of the theorem which has for its basis the following lemma concerning the approximation to a particular type of function $F(x)$.

LeMma 1. Suppose that $F(x)$ has a pth derivative which satisfies a Lipschitz condition of order $u$ with coefficient $L$ for all real values of $x$ and that $|F(x)|$ nowhere exceeds $\mu$ and is identically zero except perhaps on $\left(-l_{1}, l_{2}\right)$. Then there exists a polynomial $Q_{n}(x)$ of degree $n>p$ such that

$$
\begin{aligned}
\int_{-\infty}^{\infty} r(x)\left|F(x)-Q_{n}(x)\right|{ }^{m} d x \leqq c\left\{L^{m}\left[A\left(l_{2}+B\right) / n^{2}\right]^{m(p+u) / 2}\right. \\
\left.+\mu^{m}\left[\int_{-\infty}^{-B} r(x)(-4 x / B)^{m n} d x+\int_{A}^{\infty} r(x)(4 x / A)^{m n} d x\right]\right\}
\end{aligned}
$$

whenever $0 \leqq l_{1} \leqq B, 0<l_{2} \leqq A$ and $B \leqq A$.

Let $y=x+B=t^{2}$ and let $F(x) \equiv \phi(y) \equiv \psi(t)$. Then by Lemma 2 of $\S 6$, $\psi^{(p)}(t)$ satisfies a Lipschitz condition of order $u$ with coefficient $L\left(B+l_{2}\right)^{(p+u) / 2}$ on $-\infty<t<\infty$ so that by Theorem $\mathrm{J}$, there exists a polynomial $p_{n}(t)$ of degree $n$ such that on $-(A+B)^{1 / 2} \leqq t \leqq(A+B)^{1 / 2}$,

$$
\left|\psi(t)-p_{n}(t)\right| \leqq c L\left[\left(B+l_{2}\right)(A+B) / n^{2}\right]^{(p+u) / 2} .
$$

Take* the polynomial $Q_{n}(x)$ as

$$
Q_{n}(x) \equiv q_{n}(y) \equiv q_{n}\left(t^{2}\right) \equiv \frac{1}{2}\left[p_{n}(t)+p_{n}(-t)\right] .
$$

Since $\psi(t)$ is an even function of $t$, the error $F(x)-Q_{n}(x)$ on $(-B, A)$ which is equal to $\psi(t)-q_{n}\left(t^{2}\right)$, does not exceed in magnitude the expression on the right in (24) which in turn does not exceed $c L\left[A\left(l_{2}+B\right) / n^{2}\right]^{(p+u) / 2}$. It may be assumed as usual that the magnitude of $Q_{n}(x)$ does not exceed $2 \mu$ on $(-B, A)$ and therefore does not exceed $2 \mu(4 x / A)^{n}$ and $2 \mu(-4 x / B)^{n}$ on $(A, \infty)$ and $(-\infty,-B)$ respectively. Integration now yields the inequality of the lemma.

TheOREM 6. Under the hypotheses of this section on $r(x)$ and $f(x)$, the minimized integral $I_{m n}$ over the doubly infinite interval does not exceed a constant multiple of

$$
\begin{aligned}
{[(M(a)} & \left.+M(-b))(A(a+B))^{(p+u) / 2}\right]^{m}\left\{n^{-m(p+u)}\right. \\
& \left.+\int_{-\infty}^{-B} r(x)(-4 x / B)^{m n} d x+\int_{A}^{\infty} r(x)(4 x / A)^{m n} d x\right\}
\end{aligned}
$$

* This device was suggested by Professor J. V. Uspensky. 


$$
\begin{aligned}
& +\int_{-\infty}^{-b} r(x)\left[M(x)(-x)^{p+u}\right]^{m} d x+\int_{a}^{\infty} r(x)\left[M(x) x^{p+u}\right]^{m} d x \\
& +\left[(M(a)+M(-b))(a+b)^{p+u}\right]^{m} \int_{a}^{2 a} r(x) d x
\end{aligned}
$$

wherever $0 \leqq b \leqq B, 2 \leqq 2 a \leqq A$ and $B \leqq A$.

The minimized integral $I_{m n}$ does not exceed

$$
2^{m} \int_{-\infty}^{\infty} r(x)|f(x)-F(x)|^{m} d x+2^{m} \int_{-\infty}^{\infty} r(x)\left|F(x)-Q_{n}(x)\right|^{m} d x
$$

where $F(x)$ and the polynomial $Q_{n}(x)$ of degree $n$ are selected arbitrarily. Let $F(x)$ be defined as the function which is zero when $x$ is not on $(-b, 2 a)$, as $f(x)-V_{p}(x,-b)$ on $(-b, a)$, and on $(a, 2 a)$ as the polynomial of degree $(2 p+1)$ which agrees with $F(x)$ and its first $p$ derivatives at $x=a$ and has a root of order $(p+1)$ at $x=2 a$. Then $F(x)$ satisfies the conditions of Lemma 1 with $L=c[M(-b)+M(a)], l_{1}=b, l_{2}=2 a$, and since $a \geqq 1, \mu=c L(a+b)^{p+u}$. Let $Q_{n}(x)$ be chosen as in Lemma 1 . Then the second integral in (26) does not exceed a constant multiple of the first term in the expression (25). The first integral in (26) does not exceed a constant multiple of the remaining three terms in (25). Therefore $I_{m n}$ does not exceed the expression (25). This completes the proof of Theorem 6.

To illustrate the application of Theorem 6, consider the exponential weight function $r(x)$ equal to $\exp \left(-h|x|^{\sigma}\right)$ and $\exp \left(-h^{\prime}|x|^{\prime}\right)\left(h, h^{\prime}>0\right.$; $\left.\sigma, \sigma^{\prime}>\frac{1}{2}\right)$ for negative and non-negative values of $x$ respectively. Let $M(x)$ be equal to $\exp \left(d|x|^{i}\right)$ and $\exp \left(d^{\prime}|x|^{i^{\prime}}\right)\left(d, d^{\prime}>0 ; i<\sigma ; i^{\prime}<\sigma^{\prime}\right)$ for negative and non-negative values of $x$ respectively. When $A=c n^{1 / \sigma}, B=c n^{1 / \sigma^{\prime}}$, $a=c(\log n)^{i / \sigma}$ and $b=c(\log n)^{i^{\prime} / \sigma^{\prime}}$, with the $c$ 's suitably chosen, then it is found by use of Theorems 6 and 2 that if $\rho$ is any assigned positive number less than 1 and if $(\alpha, \beta)$ is a given finite interval, then

$$
\left|f(x)-P_{m n}(x)\right| \leqq c n^{-(1 / 2) \rho\left[2-\left(1 / \sigma+1 / \sigma^{\prime}\right)\right]+1 / m}
$$

uniformly on $(\alpha, \beta)$. This result corresponds to that of Corollary $1 \S 5$; a result corresponding to Theorem 4 may be stated; this statement as well as the details of the verification of the statements made above are left for the reader.

In conclusion, notice that the admissibility of the above skew weight function requires only that $\left(1 / \sigma+1 / \sigma^{\prime}\right)<2$ so that in the special case of an even weight function, $\sigma=\sigma^{\prime}$, it is necessary that $\sigma=\sigma^{\prime}$ exceed 1 as was the case in \$5. If $\sigma$ and $\sigma^{\prime}$ both exceed 1 and $\sigma^{\prime}<\sigma$ then the weight function is 
admissible on the basis of $\S 5$ but $\left(1 / \sigma+1 / \sigma^{\prime}\right)$ in (27) must be replaced by $\left(2 / \sigma^{\prime}\right)$ which increases the upper bound for the error essentially. If $\sigma^{\prime}$ is between $\frac{1}{2}$ and 1 , then the weight function is inadmissible on the basis of $\$ 5$ but is admissible here if $\sigma$ exceeds $\sigma^{\prime} /\left(2 \sigma^{\prime}-1\right)$; the restriction on $r(x)$ for large positive values of $x$ can thus be weakened at the expense of increasing the restriction on $r(x)$ for large negative values of $x$ and vice versa.

UNIVERSTTY OF IOWA,

Iowa Ciry, Iowa 